\title{
Further results on non-diagonal Bianchi type III vacuum metrics
}

\author{
Galliano VALENT \\ Laboratoire de Physique Théorique et des Hautes Energies \\ CNRS, UMR 7589 \\ 2 Place Jussieu, F-75251 Paris Cedex 05, France
}

\begin{abstract}
We present the derivation, for these vacuum metrics, of the Painlevé VI equation first obtained by Christodoulakis and Terzis, from the field equations for both minkowskian and euclidean signatures. This allows a complete discussion and the precise connection with some old results due to Kinnersley. The hyperkähler metrics are shown to belong to the Multi-Centre class and for the cases exhibiting an integrable geodesic flow the relevant Killing tensors are given. We conclude by the proof that for the Bianchi B family, excluding type III, there are no hyperkähler metrics.
\end{abstract}




\section{Introduction}

The study of exact solutions for empty space Einstein equations with Bianchi type B isometries has been worked out by Christodoulakis and Terzis. In a series of groundbreaking papers [3], 4], 21], [22] these authors have obtained the most general form of the mixing matrix and reduced the field equations to Painlevé VI for the types: III, IV and $\mathrm{VII}_{h}$, the more difficult type $\mathrm{VI}_{h}$ remaining still unsolved.

The special case of Bianchi type III empty space metrics was analyzed in [3] and [4]. Despite the many results obtained in these two references, some questions were left aside :

1. Their differential system, given by their equations (2.8) to (2.10) in reference [4], does solve Einstein equations. However since it was not derived from the field equations it is not equivalent to them and the analysis cannot be claimed to be fully general.

2. A new interesting euclidean metric was found while the authors were looking for minkowskian metrics. Why is it so?

3. They found several explicit minkowskian metrics of Petrov type D. What is their precise relation with Kinnersley metrics [12]?

4. Among all of the euclidean metrics which ones are hyperkähler?

It is the aim of this article to clarify these points and to study which metrics, within this family, exhibit an integrable geodesic flow.

In Section 2 we write down the field equations for both minkowskian and euclidean signatures in which a parameter $\kappa$ play a prominent role. In Section 3 the special case $\kappa=0$ is first considered leading to Kinnersley metrics and their euclidean partners. It is then possible to relate precisely all of the Petrov type D metrics found in [4] with Kinnersley's metrics. Among the euclidean metrics there is a "little" hyperkähler metric which is shown to belong to the Multi-Centre family. In Section 4 the general case, for which $\kappa \neq 0$, is discussed and it is shown that all the functions appearing in the metric can be expressed in terms of a single function $\mu(t)$ and its derivatives. It follows in Section 5 that $\mu(t)$ satisfies a non-linear second order ordinary differential equation, which can be related, using some results due to Okamoto, to Painlevé VI and which is different from the one found in [4]. Using a classical solution we retrieve the euclidean metric first discovered in [4], which we show to be hyperkähler and it reduces, in some limit, to the "little" hyperkähler metric. In Section 6 the case of a minkowskian signature singles out a parameter $E$, and only for $E \neq 0$ do we get Painlevé VI, while for the special value $E=0$ a Lie symmetry allows for integration and gives Siklos metric. In Section 7 the metrics with an integrable geodesic flow are determined and their (quadratic) Killing-Stäckel tensor is constructed. Eventually, in Section 8, it is proved that for the Bianchi B family, except for type III, there can be no hyperkähler metric.

\section{Derivation of the field equations}

For the Bianchi type III Lie algebra the Maurer-Cartan 1-forms are:

$$
\sigma_{1}=d x, \quad \sigma_{2}=d y, \quad \sigma_{3}=e^{-x} d z \quad \Rightarrow \quad d \sigma_{1}=d \sigma_{2}=0, \quad d \sigma_{3}=\sigma_{3} \wedge \sigma_{1},
$$


with the Killing fields and non-vanishing commutator

$$
\mathcal{L}_{1}=\partial_{x}+z \partial_{z}, \quad \mathcal{L}_{2}=\partial_{y}, \quad \mathcal{L}_{3}=\partial_{z} \quad\left[\mathcal{L}_{3}, \mathcal{L}_{1}\right]=\mathcal{L}_{3} .
$$

As shown in [4] the most general non-diagonal metric allows for a mixing of the $\sigma_{2}$ and $\sigma_{3}$ forms. We found convenient to write the metric

$$
g=\epsilon \alpha^{2} d t^{2}+\beta^{2} \sigma_{1}^{2}+\gamma^{2} \sigma_{3}^{2}+\delta^{2}\left(\sigma_{2}+\mu \sigma_{3}\right)^{2}, \quad \epsilon= \pm 1,
$$

where all the functions involved depend solely on $t$.

We will follow Geroch analysis [8] with respect to the Killing vector $\mathcal{L}_{2}=\partial_{y}$ and write the metric

$$
g=\frac{1}{V}(d y+\Theta)^{2}+V \Gamma
$$

with

$$
\left\{\begin{array}{l}
V=\frac{1}{\delta^{2}} \quad \Theta=\mu e^{-x} d z \\
\Gamma=\epsilon \alpha^{2} \delta^{2} d t^{2}+\beta^{2} \delta^{2} d x^{2}+\gamma^{2} \delta^{2} \sigma_{3}^{2}=e_{1}^{2}+e_{2}^{2}+e_{3}^{2} .
\end{array}\right.
$$

The empty space Einstein equations are equivalent to

$$
\begin{aligned}
& \text { (a) } *_{\Gamma} \frac{d \Theta}{V^{2}}=d \Psi, \\
& \text { (b) } \quad r i c_{i j}(\Gamma)=\frac{V^{2}}{4}\left(\partial_{i} \chi_{+} \partial_{j} \chi_{-}+\partial_{j} \chi_{+} \partial_{i} \chi_{-}\right), \quad \chi_{ \pm}=\frac{1}{V} \pm \Psi, \\
& \text { (c) } \frac{\Delta V}{V}=-2 r(\Gamma),
\end{aligned}
$$

where $\operatorname{ric}_{i j}(\Gamma)$ and $r(\Gamma)$ are the Ricci tensor and the scalar curvature of the metric $\Gamma$. An easy computation gives

$$
d \Psi=*_{\Gamma} \frac{d \Theta}{V^{2}}=-\frac{\dot{\mu} \beta \delta^{3}}{\alpha \gamma} d x-\frac{\mu \alpha \delta^{3}}{\beta \gamma} d t
$$

and its integrability condition is nothing but

$$
\dot{\mu}=\kappa \frac{\alpha \gamma}{\beta \delta^{3}} \Rightarrow \partial_{x} \Psi=-\kappa \quad \partial_{t} \Psi=-\frac{\mu \alpha \delta^{3}}{\beta \gamma}
$$

where $\kappa$ is some real constant.

The non-diagonal equation for $r i c_{i j}(\Gamma)$ gives a first order relation, which we collect with (5):

$$
\frac{\dot{\beta}}{\beta}=\frac{\dot{\gamma}}{\gamma}+\frac{\kappa}{2} \frac{\alpha \mu}{\beta \gamma \delta}, \quad \dot{\mu}=\kappa \frac{\alpha \gamma}{\beta \delta^{3}} .
$$

The remaining ones, after taking some combinations, are most conveniently written

$$
\begin{aligned}
& \frac{\ddot{\beta}}{\beta}+\frac{\dot{\beta}}{\beta}\left(\frac{\dot{\gamma}}{\gamma}+\frac{\dot{\delta}}{\delta}-\frac{\dot{\alpha}}{\alpha}\right)+\epsilon \frac{\alpha^{2}}{\beta^{2}}+\frac{\epsilon}{2} \frac{\alpha^{2} \delta^{2} \mu^{2}}{\beta^{2} \gamma^{2}}=0 \\
& \frac{(\dot{\gamma} \delta)}{\gamma \delta}+\frac{(\dot{\gamma} \delta)}{\gamma \delta}\left(\frac{\dot{\beta}}{\beta}-\frac{\dot{\alpha}}{\alpha}\right)+\epsilon \frac{\alpha^{2}}{\beta^{2}}=0 \\
& 2\left(\frac{\dot{\beta} \dot{\gamma}}{\beta \gamma}+\frac{\dot{\gamma} \dot{\delta}}{\gamma \delta}+\frac{\dot{\delta} \dot{\beta}}{\delta \beta}\right)+2 \epsilon \frac{\alpha^{2}}{\beta^{2}}+\frac{\epsilon}{2} \frac{\alpha^{2} \delta^{2} \mu^{2}}{\beta^{2} \gamma^{2}}-\frac{\kappa^{2}}{2} \frac{\alpha^{2}}{\beta^{2} \delta^{4}}=0
\end{aligned}
$$


Let us notice that the differential equation for $\gamma$, which is

$$
\frac{\ddot{\gamma}}{\gamma}+\frac{\dot{\gamma}}{\gamma}\left(\frac{\dot{\beta}}{\beta}+\frac{\dot{\delta}}{\delta}-\frac{\dot{\alpha}}{\alpha}\right)+\epsilon \frac{\alpha^{2}}{\beta^{2}}+\frac{\epsilon}{2} \frac{\alpha^{2} \delta^{2} \mu^{2}}{\beta^{2} \gamma^{2}}+\frac{\kappa^{2}}{2} \frac{\alpha^{2}}{\beta^{2} \delta^{4}}=0
$$

follows from (77(a)) and the first relation in (6) .

\section{The special case $\kappa=0$}

Since this case should correspond to Kinnersley metric let us derive it shortly. The function $\mu=\mu_{0}$ is constant, and (5) implies $\beta=c \gamma$ for some constant $c \neq 0$. Taking $\alpha=1 / \delta$ to fix up the time coordinate, equation $(17(b))$ gives

$$
\gamma \delta(\ddot{\gamma} \delta)+(\dot{\gamma} \delta)^{2}+\frac{\epsilon}{c^{2}}=0 \Rightarrow(\gamma \delta)^{2}=a-2 m t-\frac{\epsilon}{c^{2}} t^{2},
$$

where $a, m$ are integration constants. The equation (8) becomes

$$
D_{t}\left(\frac{\dot{\gamma}}{\gamma}\right)+2 \frac{\dot{\gamma}^{2}}{\gamma^{2}}+2 \frac{\dot{\gamma}}{\gamma} \frac{\dot{\delta}}{\delta}+\frac{\epsilon \mu_{0}^{2}}{2} \frac{1}{c^{2} \gamma^{4}}+\frac{\epsilon}{c^{2} \gamma^{2} \delta^{2}}=0
$$

and relation $(7(\mathrm{c}))$

$$
\frac{\dot{\gamma}^{2}}{\gamma^{2}}+2 \frac{\dot{\gamma}}{\gamma} \frac{\dot{\delta}}{\delta}+\frac{\epsilon \mu_{0}^{2}}{4} \frac{1}{c^{2} \gamma^{4}}+\frac{\epsilon}{c^{2} \gamma^{2} \delta^{2}}=0
$$

Subtracting these relations we obtain

$$
\ddot{\gamma}+\frac{\epsilon \mu_{0}^{2}}{4} \frac{1}{\gamma^{3}}=0 \Rightarrow \dot{\gamma}^{2}=E+\frac{\epsilon \mu_{0}^{2}}{4 c^{2}} \frac{1}{\gamma^{2}} .
$$

\subsection{Minkowskian signature and Kinnersley metric}

For $\epsilon=-1$ we must have $E>0$. One gets

$$
\gamma^{2}=E\left(t^{2}+\frac{\mu_{0}^{2}}{4 c^{2} E^{2}}\right)
$$

and imposing (9) we have

$$
c^{2}=1 \quad a=-\frac{\mu_{0}^{2}}{4 E^{2}}
$$

So it is convenient to define

$$
l=\frac{\mu_{0}}{2 E} \quad \Rightarrow \quad \gamma^{2}=E\left(t^{2}+l^{2}\right)
$$

and, up to scalings, we get the metric

$$
\frac{g}{E}=-\frac{d t^{2}}{U}+\left(t^{2}+l^{2}\right)\left(\sigma_{1}^{2}+\sigma_{3}^{2}\right)+U\left(\sigma_{2}+2 l \sigma_{3}\right)^{2} \quad U=\frac{t^{2}-2 m t-l^{2}}{t^{2}+l^{2}}
$$

which is Petrov type D. Since all the type D vacuum metrics were classified by Kinnersley, let us give the precise relation to his work. 
Let us consider specifically [12] his II-D metric. Up to slight notational changes one has

$$
\begin{aligned}
g=-\frac{\rho}{\Delta} d t^{2}+\frac{\Delta}{\rho}\left(d u+\left(2 l e^{-x}+a e^{-2 x}\right)\right. & \left.d v-\frac{\rho}{\Delta} d t\right)^{2} \\
& +\rho d x^{2}+\frac{e^{-2 x}}{\rho}\left(a d u-\left(t^{2}+l^{2}\right) d v\right)^{2}
\end{aligned}
$$

with

$$
\Delta=t^{2}-2 m t-l^{2}, \quad \rho=t^{2}+\left(l+a e^{-x}\right)^{2} .
$$

The transformations

$$
d y=d u-\frac{\left(t^{2}+l^{2}\right)}{\Delta} d t, \quad d z=d v-\frac{a}{\Delta} d t
$$

bring (12) to a Boyer-Lindquist form

$$
g=-\frac{\rho}{\Delta} d t^{2}+\frac{\Delta}{\rho}\left(d y+\left(2 l e^{-x}+a e^{-2 x}\right) d z\right)^{2}+\rho d x^{2}+\frac{e^{-2 x}}{\rho}\left(a d y-\left(t^{2}+l^{2}\right) d z\right)^{2} .
$$

This type $\mathrm{D}$ metric has only two commuting Killing vectors $\partial_{y}$ and $\partial_{z}$ but, as observed by [1], in the limit $a \rightarrow 0$, there appear 4 Killing vectors, and the metric (13) does transform into (11). The Bianchi type III isometries are manifest and an extra fourth Killing vector

$$
\mathcal{L}_{4}=z \partial_{x}+2 l e^{x} \partial_{y}+\frac{1}{2}\left(z^{2}-e^{2 x}\right) \partial_{z}
$$

leaves the metric invariant because of the relations

$$
\mathcal{L}_{4} \sigma_{2}=2 l e^{x} \sigma_{1} \quad \mathcal{L}_{4} \sigma_{3}=-e^{x} \sigma_{1} \quad \mathcal{L}_{4} \sigma_{1}=+e^{x} \sigma_{3}
$$

These isometries have for non-vanishing commutators

$$
\left[\mathcal{L}_{3}, \mathcal{L}_{1}\right]=\mathcal{L}_{3}, \quad\left[\mathcal{L}_{1}, \mathcal{L}_{4}\right]=\mathcal{L}_{4} \quad\left[\mathcal{L}_{3}, \mathcal{L}_{4}\right]=\mathcal{L}_{1}
$$

So we do realize that Kinnersley had discovered in 1969 the first non-diagonal Bianchi type III metric!

The limit $l \rightarrow 0$ allows, by a scaling of $t$, to take $2 m=1$ and gives the diagonal metric

$$
g_{0}=-\frac{d t^{2}}{1-1 / t}+t^{2}\left(\sigma_{1}^{2}+\sigma_{3}^{2}\right)+\left(1-\frac{1}{t}\right) \sigma_{2}^{2},
$$

which was derived just a year before by Stewart and Ellis [19] and by Cahen and Defrise [2] and re-discovered later in [15] and [14].

As observed by Christodoulakis and Terzis some of their metrics [4] being Petrov type D should be special cases of Kinnersley. A first example is given in their formula (2.37). Using the relation $B=C+4 A$ and up to a change of the normalization of $\sigma_{3}$ we get

$$
g_{1}=-A d \xi^{2}+A\left(\sigma_{1}^{2}+\sigma_{3}^{2}\right)+C\left(\sigma_{2}+\frac{1}{2} \sigma_{3}\right)^{2}
$$


with

$$
\left.A=\frac{1}{2}\left(\cosh ^{2} \xi+2 \lambda \cosh \xi+1\right), \quad C=\frac{4\left(1-\lambda^{2}\right) \sinh ^{2} \xi}{A}, \quad \lambda \in\right]-1,+1[.
$$

The change of variable and of parameter

$$
\cosh \xi=\frac{t-m}{\sqrt{m^{2}+l^{2}}}, \quad \lambda=\frac{m}{\sqrt{m^{2}+l^{2}}}, \quad l=\frac{1}{4}
$$

shows that $g_{1}$ is homothetic to Kinnersley metric (11) for the special value of $l$ given above.

A second example is their metric:

$$
g_{2}=-\frac{e^{2 \xi}\left(e^{2 \xi}+1\right)}{4\left(2 e^{2 \xi}+1\right)} d \xi^{2}+\frac{e^{\xi} \cosh \xi}{4}\left(\sigma_{1}^{2}+\sigma_{3}^{2}\right)+\frac{e^{\xi}}{2 \cosh \xi}\left(\sigma_{2}+\frac{1}{2} \sigma_{3}\right)^{2} .
$$

The change of variable and the choice of parameters

$$
e^{\xi}=2 \sqrt{2\left(t^{2}-1 / 16\right)}, \quad m=0, \quad l=\frac{1}{4},
$$

show that $g_{2}$ is again Kinnersley metric (11) for the special values of $(m, l)$ given above. Let us consider now the euclidean signature.

\subsection{Euclidean signature}

For $\epsilon=+1$, according to the sign of $E$, the metrics are:

$$
\begin{array}{lll}
E>0 & g_{+}=\frac{d t^{2}}{U_{+}}+\left(t^{2}-l^{2}\right)\left(\sigma_{1}^{2}+\sigma_{3}^{2}\right)+U_{+}\left(\sigma_{2}+2 l \sigma_{3}\right)^{2} & U_{+}=-\frac{t^{2}-2 m t+l^{2}}{t^{2}-l^{2}} \\
E=0 & g_{0}=\frac{d t^{2}}{U_{0}}+t\left(\sigma_{1}^{2}+\sigma_{3}^{2}\right)+U_{0}\left(\sigma_{2}+\sigma_{3}\right)^{2} & U_{0}=\frac{a^{2}-t^{2}}{t} \\
E<0 & g_{-}=\frac{d t^{2}}{U_{-}}+\left(l^{2}-t^{2}\right)\left(\sigma_{1}^{2}+\sigma_{3}^{2}\right)+U_{-}\left(\sigma_{2}+2 l \sigma_{3}\right)^{2} & U_{-}=-\frac{t^{2}-2 m t+l^{2}}{l^{2}-t^{2}}
\end{array}
$$

They all exhibit a fourth Killing vector given by (14). Let us also notice that despite the relation $g_{+}=-g_{-}$the two metrics for $E \neq 0$ will be different since positivity will give definitely different intervals of variation for $t$.

We will call the metric for $E=0$ the "little" hyperkähler metric since it is a special case of the more general hyperkähler metric that will be discussed later in Section 5 .

Let us prove :

Proposition 1 The metric in (19) for $E=0$ is hyperkähler (or with self-dual curvature) since it belongs to the Multi-Centre family.

Proof: The reader may consult [24] for the definition and some basic properties of the Multi-Centre metrics. Using for preferred Killing $\partial_{z}$ the metric becomes

$$
g=\frac{1}{V}(d z+\Theta)^{2}+V \Gamma
$$


with

$$
V=\frac{t}{a^{2} X^{2}} \quad \Theta=\frac{a^{2}-t^{2}}{a^{2} X} d y \quad X=e^{-x}
$$

and the three dimensional metric

$$
\Gamma=a^{2}\left\{d X^{2}+\frac{X^{2}}{\left(a^{2}-t^{2}\right)} d t^{2}+X^{2} \frac{\left(a^{2}-t^{2}\right)}{a^{2}} d y^{2}\right\}=d u^{2}+d v^{2}+d w^{2}
$$

with the flattening coordinates

$$
u=X \sqrt{a^{2}-t^{2}} \cos y \quad v=X \sqrt{a^{2}-t^{2}} \sin y \quad w=X t .
$$

The potential and the gauge field become

$$
V=a \frac{w}{r^{3}} \quad \Theta=-a \frac{(v d u-u d v)}{r^{3}} \quad r=\sqrt{u^{2}+v^{2}+w^{2}} .
$$

The potential is that of a dipole located at the origin and aligned with the $w$ axis. It obviously satisfies $\Delta V=0$ proving that the metric is indeed a Multi-Centre, hence hyperkähler. The triplet of covariantly constant complex structures $\Omega_{i}$ is

$$
\left\{\begin{array}{l}
\Omega_{1}=(d z+\Theta) \wedge d u+V d v \wedge d w \\
\Omega_{2}=(d z+\Theta) \wedge d v+V d w \wedge d u \\
\Omega_{3}=(d z+\Theta) \wedge d w+V d u \wedge d v
\end{array}\right.
$$

and this ends the proof.

The Killing vectors, in these coordinates, become

$$
\begin{aligned}
& \mathcal{L}_{1}=z \partial_{z}-u \partial_{u}-v \partial_{v}-w \partial_{w} \quad \mathcal{L}_{2}=-v \partial_{u}+u \partial_{v} \quad \mathcal{L}_{3}=\partial_{z} \\
& \mathcal{L}_{4}=\frac{1}{2}\left(z^{2}-\frac{a^{2}}{r^{2}}\right) \partial_{z}-\left(z u+a \frac{v}{r}\right) \partial_{u}-\left(z v-a \frac{u}{r}\right) \partial_{v}-z w \partial_{w} .
\end{aligned}
$$

It follows that $\mathcal{L}_{1}, \mathcal{L}_{3}, \mathcal{L}_{4}$ are tri-holomorphic

$$
\mathcal{L}_{a} \Omega_{i}=0 \quad a=1,3,4 \quad i=1,2,3
$$

while $\mathcal{L}_{2}$ is holomorphic

$$
\mathcal{L}_{2} \Omega_{1}=-\Omega_{2} \quad \mathcal{L}_{2} \Omega_{2}=\Omega_{1} \quad \mathcal{L}_{2} \Omega_{3}=0 .
$$

Let us consider now the general case for which $\kappa \neq 0$.

\section{General case}

A convenient choice of time coordinate is $\kappa \alpha=\beta \gamma \delta$ which simplifies the two relations in (6) to

$$
\dot{\mu}=\frac{\gamma^{2}}{\delta^{2}}>0 \quad \frac{\mu}{2}=\frac{\dot{\beta}}{\beta}-\frac{\dot{\gamma}}{\gamma}
$$


and allows to express all functions in terms of $\gamma \delta, \mu$ and their derivatives:

$$
\gamma^{2}=\gamma \delta \sqrt{\dot{\mu}} \quad \delta^{2}=\frac{\gamma \delta}{\sqrt{\dot{\mu}}} \quad 2 \frac{\dot{\beta}}{\beta}=\frac{\ddot{\mu}}{2 \dot{\mu}}+\mu+\frac{(\dot{\gamma} \delta)}{\gamma \delta} \quad \alpha^{2}=\beta^{2} \frac{(\gamma \delta)^{2}}{\kappa^{2}} .
$$

By a scaling on $\gamma$ and $\delta$ we can set $\kappa=1$ and equation $(7(b))$ which becomes

$$
D_{t}\left(\frac{(\dot{\gamma} \delta)}{\gamma \delta}\right)+\epsilon(\gamma \delta)^{2}=0
$$

can be integrated once. So we are left with the differential system:

$$
\begin{array}{lll}
\text { (a) } & \left(\frac{(\dot{\gamma} \delta)}{\gamma \delta}\right)^{2}+\epsilon(\gamma \delta)^{2}=E & \dot{\mu}>0 \\
\text { (b) } \quad & \left(\frac{\ddot{\mu}}{2 \dot{\mu}}-\frac{(\dot{\gamma} \delta)}{\gamma \delta}\right)^{2}+\dot{\mu}-2 \frac{(\dot{\gamma} \delta)}{\gamma \delta} \mu-4 E-\epsilon(\gamma \delta)^{2} \frac{\mu^{2}}{\dot{\mu}}=0 &
\end{array}
$$

where $E$ is some real constant. Let us notice that equation (7)(a)), formerly the differential equation for $\beta$, becomes

$$
D_{t} A+\dot{\mu}+\epsilon(\gamma \delta)^{2} \frac{\mu^{2}}{\dot{\mu}}=0 \quad A=\frac{\ddot{\mu}}{2 \dot{\mu}}-\frac{(\dot{\gamma} \delta)}{\gamma \delta}
$$

and should appear as equation (28(c)). However differentiating (28(b)) we get

$$
A\left(D_{t} A+\dot{\mu}+\epsilon(\gamma \delta)^{2} \frac{\mu^{2}}{\dot{\mu}}\right)=0
$$

Since the relations in (29) imply that $A$ cannot vanish identically, the equation (29) is a consequence of (28(b)), showing that this last equation is an integrated form of (29).

Let us first consider the euclidean signature.

\section{Euclidean signature}

In this case $E>0$, and the general solution of $(28(\mathrm{a}))$ can be written

$$
\gamma \delta=\frac{\sqrt{E}}{\cosh (\sqrt{E} t)}
$$

By the scalings $\mu \rightarrow \mu / \sqrt{E}$ and $t \rightarrow \sqrt{E} t$ we can take $E=1$. The ode $(28(\mathrm{~b}))$ becomes

$$
\left(\frac{\ddot{\mu}}{2 \dot{\mu}}+\tanh t\right)^{2}+\dot{\mu}+2 \tanh t \mu-4-\left(1-\tanh ^{2} t\right) \frac{\mu^{2}}{\dot{\mu}}=0 \quad \dot{\mu}>0
$$

and the metric

$$
g=\beta^{2}\left(\frac{d t^{2}}{\cosh ^{2} t}+\sigma_{1}^{2}\right)+\frac{\sqrt{\dot{\mu}}}{\cosh t} \sigma_{3}^{2}+\frac{1}{\cosh t \sqrt{\dot{\mu}}}\left(\sigma_{2}+\mu \sigma_{3}\right)^{2}
$$


with

$$
\frac{\left(\dot{\beta}^{2}\right)}{\beta^{2}}=\frac{\ddot{\mu}}{2 \dot{\mu}}+\mu-\tanh t .
$$

It is convenient to make the transformations

$$
x=(1+\tanh t) / 2 \quad \quad \quad \quad m^{\prime}=D_{x} m
$$

which bring the ode (32) into

$$
x^{2}(1-x)^{2}\left(m^{\prime \prime}\right)^{2}=-4 m^{\prime}\left(x m^{\prime}-m\right)^{2}+4 m^{\prime 2}\left(x m^{\prime}-m\right)+4 m^{\prime 2}, \quad x \in(0,1) .
$$

This ODE is clearly different from the one obtained by Christodoulakis and Terzis in [6]. In their work they used results of Cosgrove and Scoufis [6] to reduce (35) to Painlevé VI. We will not follow this path since Okamoto [17] gave much simpler Bäcklund transformations. Using Okamoto notations let us take $b_{2}=0$ and denote $\mathbf{b}=\left(b_{1}, b_{3}, b_{4}\right)$. Let us consider the solution $y(x, \mathbf{b})$ of Painlevé VI with parameters

$$
\alpha=\frac{1}{2}\left(b_{3}-b_{4}\right)^{2} \quad \beta=-\frac{1}{2} b_{1}^{2} \quad \gamma=\frac{1}{2} b_{1}^{2} \quad \delta=\frac{1}{2}\left[1-\left(b_{3}+b_{4}+1\right)^{2}\right] .
$$

If one defines

$$
h(x)=x(x-1) H(x)+s_{2}(\mathbf{b})\left(x-\frac{1}{2}\right),
$$

where $H$ is the Hamilton function

$$
\left\{\begin{array}{c}
x(x-1) H=y(y-1)(y-x) z^{2}-\left[b_{1}(2 y-1)(y-x)+\left(b_{3}+b_{4}\right) y(y-1)\right] z \\
+\left(b_{1}+b_{3}\right)\left(b_{1}+b_{4}\right)(y-x) \\
2 z=\frac{x(x-1) y^{\prime}}{y(y-1)(y-x)}+\frac{b_{1}(2 y-1)}{y(y-1)}+\frac{b_{3}+b_{4}}{y-x}
\end{array}\right.
$$

then $h$ is a solution of

$$
x^{2}(x-1)^{2}\left(h^{\prime \prime}\right)^{2}=-4 h^{\prime}\left(x h^{\prime}-h\right)^{2}+4\left(h^{\prime}\right)^{2}\left(x h^{\prime}-h\right)+s_{1}\left(\mathbf{b}^{2}\right)\left(h^{\prime}\right)^{2}+s_{2}\left(\mathbf{b}^{2}\right) h^{\prime}+s_{3}\left(\mathbf{b}^{2}\right)
$$

where the $s_{i}\left(\mathbf{b}^{2}\right) i=1,2,3$ are the symmetric polynomials for $\mathbf{b}^{2}=\left(b_{1}^{2}, b_{3}^{2}, b_{4}^{2}\right)$. It follows that $m=h$ is a solution of (35).

The identification of (38) and (35) gives 3 different cases:

$$
\begin{array}{ccc} 
& \left(b_{1}, b_{3}, b_{4}\right) & (\alpha, \beta, \gamma, \delta) \\
(a) & (0,+2,0) & (2,0,0,-4) \\
(b) & (0,-2,0) & (2,0,0,0) \\
(c) & (2,0,0) & (0,-2,2,0)
\end{array}
$$

The Bäcklund transformation follows from (37); for the first two cases

$$
\text { (a) } \quad(b) \quad \rightarrow \quad m=\frac{1}{4 y(y-1)(y-x)^{2}}\left\{x^{2}(x-1)^{2}\left(y^{\prime}\right)^{2}-4 y^{2}(y-1)^{2}\right\}
$$


while for the third case

$$
\text { (c) } \rightarrow \quad m=\frac{1}{4 y(y-1)(y-x)^{2}}\left\{x^{2}(x-1)^{2}\left(y^{\prime}\right)^{2}-4(y-x)^{2}\right\}
$$

The derivative of $m$ is

$$
\begin{aligned}
& \text { (a) } \rightarrow \quad m^{\prime}=-\frac{1}{4 y(y-1)}\left[\frac{x(x-1)\left(y^{\prime}\right)^{2}+2 y(y-1)}{(y-x)}\right]^{2} \\
& \text { (b) } \rightarrow \quad m^{\prime}=-\frac{1}{4 y(y-1)}\left[\frac{x(x-1)\left(y^{\prime}\right)^{2}-2 y(y-1)}{(y-x)}\right]^{2} \\
& \text { (c) } \rightarrow \quad m^{\prime}=-\frac{m}{y-x}
\end{aligned}
$$

so there are are further restrictions on $y$ since $m^{\prime}$ must be negative.

\section{Remarks:}

1. Using the birational transformations given by Okamoto, all of these solutions can be reduced to $\mathbf{b}=(0,0,0)$ which means that $(\alpha, \beta, \gamma, \delta)=(0,0,0,0)$. Despite the great simplicity of the parameters the complete solution is not known.

2. In the first two cases there are "classical solutions" given by

$$
\text { (a) : } y=\frac{(1-x)^{2}}{(1-x)^{2}+C x^{2}} \quad(b): \quad y=\frac{x^{2}}{2 x-1+C(1-x)^{2}},
$$

but their Bäcklund transform (42) gives $m \equiv 0$.

3. In the last two cases there are also classical solutions given by [10]. Only for case (b) do we get the non-trivial result

$$
\frac{m}{2}=\frac{2 x-1+2 x(1-x) F}{1+x(1-x) F^{2}} \quad F=\ln \left(\frac{x}{1-x}\right)+2 a, \quad a \in \mathbb{R} .
$$

Going back to the $t$ variable it is convenient to define

$$
U=(t+a) \tanh t-1 \quad a \in \mathbb{R} \quad \Longrightarrow \quad \ddot{U}+\frac{2}{\cosh ^{2} t} U=0,
$$

which allows to write

$$
\frac{\mu}{2}=\frac{\dot{U}}{\dot{U}^{2}+U^{2} / \cosh ^{2} t} \quad \frac{\dot{\mu}}{4}=\frac{U^{2} / \cosh ^{2} t}{\left(\dot{U}^{2}+U^{2} / \cosh ^{2} t\right)^{2}},
$$

and upon integration of relations (26) one gets (up to scalings) the final form of this euclidean metric

$$
g=U\left(\frac{d t^{2}}{\cosh ^{2} t}+\sigma_{1}^{2}\right)+\frac{U}{\cosh ^{2} t} \sigma_{2}^{2}+\frac{1}{U}\left(\sigma_{3}+\dot{U} \sigma_{2}\right)^{2} .
$$

Let us observe that in the limit $a \rightarrow+\infty$, dividing the full metric by $a$ and changing $\sigma_{3} \rightarrow \sigma_{3} / a$ one gets

$$
g_{\infty}=\frac{\tanh t}{\cosh ^{2} t} d t^{2}+\tanh t\left(\sigma_{1}^{2}+\sigma_{3}^{2}\right)+\frac{\cosh ^{2} t}{\tanh t}\left(\sigma_{2}+\sigma_{3}\right)^{2}
$$


and upon the change of coordinate $u=a \tanh t$ one recovers the metric (19) for $E=0$ which was already proved to be hyperkähler.

In fact the euclidean metric (46) is nothing but Christodoulakis and Terzis metric [4] written as:

$$
A d \xi^{2}+B \sigma_{1}^{2}+D \sigma_{2}^{2}+2 C \sigma_{2} \sigma_{3}+\frac{1}{B} \sigma_{3}^{2}
$$

where all functions depend solely on $\xi$. Noticing the relation

$$
\frac{1}{B}-\frac{C^{2}}{D}=\frac{1}{\cosh ^{2}\left(\mu^{2} \cos ^{2} \xi\right) D}
$$

and using the variable $t=\mu^{2} \cos ^{2} \xi$, some algebra and several scalings bring the metric (47) to the form (46).

Let us prove:

Proposition 2 The metric (46) is hyperkähler since it belongs to the Multi-Centre family.

Proof: We will use the Killing vector $\partial_{z}$ to write (46) in the Multi-Centre form

$$
g=\frac{1}{V}(d z+\Theta)^{2}+V \Gamma
$$

with

$$
X=e^{-x} \quad \frac{1}{V}=\frac{X^{2}}{U} \quad \Theta=\frac{\dot{U}}{X} d y
$$

and the three dimensional metric

$$
\Gamma=d X^{2}+\frac{X^{2}}{\cosh ^{2} t}\left(d t^{2}+d y^{2}\right)=d u^{2}+d v^{2}+d w^{2}
$$

with the flattening coordinates

$$
u=\frac{X}{\cosh t} \cos y \quad v=\frac{X}{\cosh t} \sin y \quad w=X \tanh t
$$

The potential becomes

$$
V=a \frac{w}{r^{3}}-\frac{1}{r^{2}}+\frac{w}{2 r^{3}} \ln \frac{r+w}{r-w}, \quad r=\sqrt{u^{2}+v^{2}+w^{2}} .
$$

We have checked that $\Delta V=0$ establishing that this metric is a Multi-Centre and hence is hyperkähler. The complex structures are still given by (24). This ends the proof.

Let us consider now the minkowskian signature.

\section{Minkowskian signature}

Now the final integration of equation (28)(a)) does depend on the sign of $E$. We must analyze separately each case. 


\subsection{First case: $\mathrm{E}>0$}

We have

$$
\frac{\gamma \delta}{\kappa}=\frac{\sqrt{E}}{\sinh (\sqrt{E} t)} .
$$

Up to scalings of $\mu$ and $t$ we can take $E=1$. The ode $(28(\mathrm{~b}))$ becomes

$$
\left(\frac{\ddot{\mu}}{2 \dot{\mu}}+\operatorname{coth} t\right)^{2}+\dot{\mu}+2 \operatorname{coth} t \mu-4+\left(\operatorname{coth}^{2} t-1\right) \frac{\mu^{2}}{\dot{\mu}}=0
$$

and the metric

$$
g=\beta^{2}\left(\frac{d t^{2}}{\sinh ^{2} t}+\sigma_{1}^{2}\right)+\frac{\sqrt{\dot{\mu}}}{\sinh t} \sigma_{3}^{2}+\frac{1}{\sinh t \sqrt{\dot{\mu}}}\left(\sigma_{2}+\mu \sigma_{3}\right)^{2}
$$

with

$$
\frac{\left(\dot{\beta}^{2}\right)}{\beta^{2}}=\frac{\ddot{\mu}}{2 \dot{\mu}}+\mu-\operatorname{coth} t .
$$

It is convenient to make the changes

$$
x=\frac{1}{2}(\operatorname{coth} t+1) \quad \mu(t)=-2 m(x) \quad m^{\prime}=D_{x} m .
$$

The equation (54) becomes

$$
x^{2}(x-1)^{2}\left(m^{\prime \prime}\right)^{2}=-4 m^{\prime}\left(x m^{\prime}-m\right)^{2}+4 m^{\prime 2}\left(x m^{\prime}-m\right)+4 m^{\prime 2}, \quad x \in(1,+\infty) .
$$

This ode is exactly the same as for the euclidean signature (compare with (35)), the main difference lies in the range for $x$ : in the euclidean case one has $x \in(0,1)$ while for the minkowskian case one has $x \in(1,+\infty)$. This explains why in [4] the authors could get also an explicit euclidean metric in despite of the fact that they were looking for minkowskian metrics.

The subsequent discussion is exactly the same as in section 5: one has three cases for the parameters and the Bäcklund transformations are the same but the sign fo $m^{\prime}$ must be positive.

An interesting question remains: what about the solution (43)? It is of course still valid if we change $1-x \rightarrow x-1$ inside the logarithm. One is led to define

$$
U=\frac{(t+a)}{\tanh t}-1 \quad a \in \mathbb{R} \quad \Longrightarrow \quad \ddot{U}-\frac{2}{\sinh ^{2} t} U=0
$$

which allows to write

$$
\frac{\mu}{2}=\frac{\dot{U}}{\dot{U}^{2}-U^{2} / \sinh ^{2} t} \quad \frac{\dot{\mu}}{4}=-\frac{U^{2} / \sinh ^{2} t}{\left(\dot{U}^{2}-U^{2} / \sinh ^{2} t\right)^{2}},
$$

showing that the positivity constraint for $\dot{\mu}$ is not valid and so we must reject this solution. This case is meaningful only for the euclidean signature, in which case the metric is hyperkähler, but does not give anything new for the minkowskian signature. 


\subsection{Second case: $E=0$}

We have

$$
\gamma \delta=\frac{1}{t} \quad \text { and } \quad\left(\frac{\ddot{\mu}}{2 \dot{\mu}}+\frac{1}{t}\right)^{2}+\dot{\mu}+\frac{2 \mu}{t}+\frac{\mu^{2}}{t^{2} \dot{\mu}}=0 .
$$

This case is again special and does not reduce to Painlevé VI because it exhibits the Lie symmetry

$$
\mu \rightarrow a \mu \quad t \rightarrow \frac{t}{a} \quad a \neq 0 .
$$

Defining the two invariants

$$
u=t \mu \quad v=t^{2} \dot{\mu}>0
$$

brings the ode to the form

$$
(u+v)^{2}\left[\left(\frac{d v}{d u}\right)^{2}+4 v\right]=0
$$

We get two solutions:

1. A singular one: $u+v=0$ which gives $\mu=-\frac{\mu_{0}}{t}$ which is acceptable for $\mu_{0}>0$.

2. The general solution cannot be real due to the constraint $v>0$ and must be rejected.

The remaining functions, using (26)

$$
\delta^{2}=\frac{\kappa}{\sqrt{\mu_{0}}} \quad \beta^{2}=\beta_{0}^{2} t^{-\left(\mu_{0}+2\right)}
$$

Up to scalings of $\sigma_{2}$ and $\sigma_{3}$ and the definitions

$$
\mu=\frac{2}{2+\mu_{0}} \quad \tau=t^{-\mu}
$$

we get eventually Siklos metric [16] as written in [3]

$$
\left.g_{S}=-\mu^{2} d \tau^{2}+\tau^{2} \sigma_{1}^{2}+\frac{\mu}{2(1-\mu)} \tau^{2 \mu} \sigma_{3}^{2}+\left(\sigma_{2}+\tau^{\mu} \sigma_{3}\right)^{2}, \quad \mu \in\right] 0,1[.
$$

This metric is rather special since it was proved in [3] that it descibes a pp-wave with a strong isometry enhancment up to six Killing vectors.

\subsection{Third case: $\mathrm{E}<0$}

We have

$$
\gamma \delta=\frac{\sqrt{|E|}}{\sin (\sqrt{|E|} t)} .
$$

Scalings of $\mu$ and $t$ allow to take $E=-1$. The ode $(28(b))$ is now

$$
\left(\frac{\ddot{\mu}}{2 \dot{\mu}}+\cot t\right)^{2}+\dot{\mu}+2 \cot t \mu+4+\frac{\mu^{2}}{\sin ^{2} t \dot{\mu}}=0
$$


and the metric

$$
g=\beta^{2}\left(\frac{d t^{2}}{\sin ^{2} t}+\sigma_{1}^{2}\right)+\frac{\sqrt{\dot{\mu}}}{\sin t} \sigma_{3}^{2}+\frac{1}{\sin t \sqrt{\dot{\mu}}}\left(\sigma_{2}+\mu \sigma_{3}\right)^{2}
$$

with

$$
\frac{\left(\dot{\beta}^{2}\right)}{\beta^{2}}=\frac{\ddot{\mu}}{2 \dot{\mu}}+\mu-\cot t
$$

One can still define, but this is quite formal

$$
x=\frac{1}{2}(\cot t+1) \quad \mu(t)=-2 m(x) \quad m^{\prime}=D_{x} m
$$

transforming the previous equation into

$$
x^{2}(x-1)^{2}\left(m^{\prime \prime}\right)^{2}=-4 m^{\prime}\left(x m^{\prime}-m\right)^{2}+4 m^{\prime 2}\left(x m^{\prime}-m\right)-4 m^{\prime 2} .
$$

We get once more the same structure but for a change of sign in the last term. One can use again Okamoto results but for $b_{1}^{2}=-4$ and $b_{4}^{2}=-4$ since the parameters $(\alpha, \beta, \gamma, \delta)$ become complex. For $b_{3}^{2}=-4$ we get real parameters

$$
b_{1}=b_{4}=0 \quad b_{3}= \pm 2 i \quad \alpha=0 \quad \beta=2 \quad \gamma=-2 \quad \delta=0 .
$$

The Bäcklund transformation is

$$
m=\frac{1}{4 y(y-1)(y-x)}\left(x^{2}(x-1)^{2}\left(y^{\prime}\right)^{2}+4(y-x)^{2}\right) .
$$

The classical solutions for $y$ are now complex but they are again mapped into $m \equiv 0$. Furthermore very little seems to be known on Painlevé VI when Okamoto parameters become complex.

The derivative of $m$ is

$$
m^{\prime}=-\frac{1}{4 y(y-1)} \frac{\left\{x^{2}(x-1)^{2}\left(y^{\prime}\right)^{2}+4(y-x)^{2}\right\}}{(y-x)^{2}}
$$

so there are are further restrictions on $y$ since $m^{\prime}$ must be positive.

\subsection{A Bäcklund transform}

Since the minkowskian case is now completely discussed it is time to compare with the results of [4]. Some computational work shows that their metric is

$$
g=\beta^{2}\left(-\frac{d x^{2}}{x(x-1)}+\sigma_{1}^{2}\right)+\widetilde{\gamma}^{2} \sigma_{3}^{2}+\widetilde{\delta}^{2}\left(\sigma_{2}+\widetilde{\mu} \sigma_{3}\right)^{2} \quad \beta^{2}=\frac{e^{u_{1}}}{4}
$$

As already observed for $x \in(1,+\infty)$ it is minkowskian while for $x \in(0,1)$ it is euclidean. Using their equation (2.8) one gets

$$
\frac{d}{d x} \ln \left(\beta^{2}\right)=\frac{2 x-1+y}{x(x-1)}
$$


where $y$ is a solution of

$$
x^{2}(x-1)^{2}\left(y^{\prime \prime}\right)^{2}=-4 y^{\prime}\left(x y^{\prime}-y\right)^{2}+4\left(y^{\prime}\right)^{2}\left(x y^{\prime}-y\right)+5\left(y^{\prime}\right)^{2}+4 y^{\prime} .
$$

The changes

$$
x=\frac{1}{2}(\operatorname{coth} t+1) \quad y=-\frac{\widetilde{\mu}}{2}
$$

transform (69) into

$$
\left(\frac{\ddot{\widetilde{\mu}}}{2 \dot{\tilde{\mu}}}+\operatorname{coth} t\right)^{2}+\dot{\tilde{\mu}}+2 \operatorname{coth} t \widetilde{\mu}-5+\frac{\left(\widetilde{\mu}^{2}-4\right)}{\sinh ^{2} t \dot{\tilde{\mu}}}=0
$$

Comparing then relation (68) and (26) we obtain the desired Bäcklund:

$$
\widetilde{\mu}=\frac{\ddot{\mu}}{2 \dot{\mu}}+\mu+\operatorname{coth} t
$$

Let us prove:

Proposition 3 The Bäcklund transformation (71) does transform (54) into (70).

Proof: Equation (29) gives

$$
\sinh ^{2} t \dot{\tilde{\mu}}=\frac{\mu^{2}}{\dot{\mu}}
$$

Using this relation to get rid of $\dot{\mu}$ in (54) gives a second degree equation for $\mu$, the solution of which is the inverse Bäcklund

$$
\mu=\frac{\sinh ^{2} t \dot{\tilde{\mu}}}{1+\sinh ^{2} t \dot{\tilde{\mu}}}(\tilde{\mu}-\operatorname{coth} t \pm \sqrt{\Delta}) \quad \Delta=-\dot{\widetilde{\mu}}-2 \operatorname{coth} t \tilde{\mu}+5-\frac{\left(\widetilde{\mu}^{2}-4\right)}{\sinh ^{2} t \dot{\tilde{\mu}}}
$$

Differentiating (72) and getting rid of $\mu$ using (73) we end up with

$$
\ddot{\widetilde{\mu}}+2 \operatorname{coth} t \dot{\widetilde{\mu}}= \pm 2 \dot{\widetilde{\mu}} \sqrt{\Delta}
$$

Squaring of both sides gives (70) and ends the proof.

It follows that in [4] the case $E<0$ was missing. The reason for this was explained in the introduction: the authors presented a solution of the Einstein equations without deriving it from the basic field equations as it is customary.

\section{$7 \quad$ Integrable geodesic flows}

As is well known the geodesic flow, for a manifold equipped with some metric $g$, is generated by the quadratic hamiltonian

$$
H=\frac{1}{2} g^{\mu \nu} P_{\mu} P_{\nu}
$$

and the Killing vectors $K_{i}^{\mu}$ generate linear conserved quantities

$$
K_{i}=K_{i}^{\mu} P_{\mu} \quad\left\{H, K_{i}\right\}=0 .
$$


The integrability, in Liouville sense, of the geodesic flow hinges on the existence of 4 algebraically independent quantities (one of them being the hamiltonian) which are in involution with respect to the Poisson bracket. In the metrics discussed in this work we always have two commuting Killing vectors which are suited to our aim, so we just need one extra conserved quantity in order to reach integrability.

There are two useful tensors which can help us: Killing-Yano (K-Y) and Killing-Stäckel (K-S) tensors. A K-Y tensor is defined by

$$
Y_{\mu \nu}=-Y_{\nu \mu} \quad \& \quad \nabla_{(\mu} Y_{\nu) \rho}=0
$$

while for a (quadratic) K-S tensor

$$
S_{\mu \nu}=S_{\nu \mu} \quad \& \quad \nabla_{(\mu} S_{\nu \rho)}=0
$$

A K-S tensor generates a quadratic conserved quantity

$$
\mathcal{S}=S^{\mu \nu} P_{\mu} P_{\nu} \quad\{H, \mathcal{S}\}=0
$$

while the symmetric product of two K-Y tensors (possibly a square) give a K-S tensor.

An important observation is that Liouville integrability ensures the existence of separating coordinates for the Hamilton-Jacobi equation. This helps to make life easier since Levi-Civita has given the necessary conditions for the separability of Hamilton-Jacobi, see for instance [18]. For a metric of the form (11) the Levi-Civita constraints are merely:

$$
\gamma^{2}=C \beta^{2} \quad \dot{\mu}(t)=0 .
$$

By scalings one can reduce the constant $C$ to be one, and the second constraint holds only for the metrics with $\kappa=0$. So we have the short list

1. The Kinnersley metric (11),

2. The "little" hyperkähler metric (19) for $E=0$,

3. The two euclidean metrics (19) for $E \neq 0$.

To achieve integrability we need to exhibit a quadratic Killing tensor for these metrics. In agreement with the general results [25], [5] for Petrov type D metrics we will check that they do exhibit at the same time a K-Y and a K-S tensor.

\subsection{Kinnersley metric}

The Killing vectors produce 4 conserved quantities

$$
\left\{\begin{array}{l}
K_{1}=-X P_{X}+z P_{z} \quad K_{2}=P_{y} \quad K_{3}=P_{z} \\
K_{4}=-z X P_{X}+\frac{2 l}{X} P_{y}+\frac{1}{2}\left(z^{2}-\frac{1}{X^{2}}\right) P_{z}
\end{array} \quad X=e^{-x} .\right.
$$

This metric exhibits the Killing-Yano tensor

$$
\mathcal{Y}=Y_{\mu \nu} d x^{\mu} \wedge d x^{\nu}=l d t \wedge\left(\sigma_{2}+2 l \sigma_{3}\right)-t\left(t^{2}+l^{2}\right) \sigma_{3} \wedge \sigma_{1}
$$


Its square gives the K-S tensor

$$
\mathcal{S}_{0}=S_{0}^{\mu \nu} P_{\mu} P_{\nu} \quad S_{0}^{\mu \nu}=Y^{\mu \sigma} g_{\sigma \tau} Y^{\tau \nu}
$$

but it is fully reducible since we have

$$
\mathcal{S}_{0}=l^{2} H-K_{1}^{2}-4 l^{2} K_{2}^{2}+2 K_{3} K_{4}
$$

Besides there is another quadratic K-S tensor

$$
\mathcal{S} \equiv S^{\mu \nu} P_{\mu} P_{\nu}
$$

which can be written

$$
\mathcal{S}=\frac{X^{2}}{\Delta} P_{X}^{2}-P_{t}^{2}+\frac{1}{X^{2} \Delta}\left(P_{z}-2 l X P_{y}\right)^{2}-4 \frac{\left(m t+l^{2}\right)}{\Delta}\left(H-\frac{t(t-m)}{\Delta} P_{y}^{2}\right)
$$

with $\Delta=t^{2}-2 m t-l^{2}$. We have checked the irreducibility of this K-S tensor and its invariance under all the isometries.

The Liouville integrability follows from the existence of the following 4 independent quantities

$$
\begin{array}{llll}
H & K_{2} & K_{3} & \mathcal{S}
\end{array}
$$

which are in involution for the Poisson bracket. As already mentioned the HamiltonJacobi equation does admit separation of variables, but since the metric is Ricci-flat quantum integrability is also preserved within "minimal quantization" (see [7]) leading to the separability of the Schrödinger equation as well.

\subsection{The "little" hyperkähler metric}

The analysis of which Multi-Centre metrics have an integrable geodesic flow, initiated in [9], was completed in [23]. In this last reference the dipolar potential appears as the special case of the first dipolar breaking of Taub-NUT with $m=v_{0}=0$ and $\mathcal{F}=a$. The hamiltonian is

$$
H=\frac{V}{2} P_{z}^{2}+\frac{1}{2 V}\left\{\left(P_{u}+\frac{a v}{r^{3}} P_{z}\right)^{2}+\left(P_{v}-\frac{a u}{r^{3}} P_{z}\right)^{2}+P_{w}^{2}\right\} .
$$

The Killing vectors give the conserved quantities

$$
\left\{\begin{array}{l}
K_{1}=z P_{z}-u P_{u}-v P_{v}-w P_{w} \quad K_{2}=-v P_{u}+u P_{v} \quad K_{3}=P_{z} \\
K_{4}=-z\left(u P_{u}+v P_{v}+w P_{w}\right)+\frac{1}{2}\left(z^{2}-\frac{a^{2}}{r^{2}}\right) P_{z}+\frac{a}{r}\left(u P_{v}-v P_{u}\right) .
\end{array}\right.
$$

Among the angular momentum components

$$
\vec{L}=\vec{r} \wedge \vec{P} \quad \vec{r}=(u, v, w)
$$

only $L_{3}$ is conserved, since it is an isometry. From the general structure of the K-S tensor given in [23] some work is needed to extract the special case $m=v_{0}=0$ and to express it in terms of the true momenta $P_{\mu}$. One gets

$$
\mathcal{S} \equiv S^{\mu \nu} P_{\mu} P_{\nu}=\vec{L}^{2}-2 a \frac{w}{r} H
$$

which is irreducible and invariant under the action of the four Killing vectors. 


\subsection{Euclidean Kinnersley metrics}

For the metric with $E>0$ in formula (19), the isometries are the same as for their minkowskian partner. The K-Y tensor is now

$$
\mathcal{Y}=Y_{\mu \nu} d x^{\mu} \wedge d x^{\nu}=l d t \wedge\left(\sigma_{2}+2 l \sigma_{3}\right)+t\left(t^{2}-l^{2}\right) \sigma_{3} \wedge \sigma_{1}
$$

and its square is still reducible. The K-S tensor is

$$
\mathcal{S}=-\frac{X^{2}}{\Delta} P_{X}^{2}+P_{t}^{2}-\frac{1}{X^{2} \Delta}\left(P_{z}-2 l X P_{y}\right)^{2}+4 \frac{\left(m t-l^{2}\right)}{\Delta}\left(H+\frac{t(t-m)}{\Delta} P_{y}^{2}\right) .
$$

with $\Delta=t^{2}-2 m t+l^{2}$.

It is also valid for the metric with $E<0$ since we have $g_{-}=-g_{+}$.

\subsection{A remark on Siklos metric}

From Levi-Civita conditions (80) we know that the geodesic flow cannot be integrable for this metric. However, in a recent work [1] it has been shown how to construct a K-S tensor for the pp-waves. Now it happens that all the necessary tools: the Killing vectors and an homothetic Killing vector were given in [3]. It is convenient to use null coordinates for which Siklos metric can be written

$$
g_{S}=-2 \mu d u d v+\frac{(2-\mu)}{2(1-\mu)} d y^{2}+2 u^{\mu} d y d z+u^{2 \mu} d z^{2}
$$

giving for hamiltonian

$$
\mu H=-P_{u} P_{v}+2(1-\mu) P_{y}^{2}-2(1-\mu) u^{-\mu} P_{y} P_{z}+(2-\mu) u^{-2 \mu} P_{z}^{2} .
$$

The homothetic Killing vector and the first 5 Killing vectors are

$$
\left\{\begin{array}{l}
Y^{H}=u P_{u}+v P_{v}+y P_{y}+(1-\mu) P_{z}, \quad K_{1}=u P_{u}-v P_{v}-\mu z P_{z}, \\
K_{2}=P_{y} \quad K_{3}=P_{z} \quad K_{4}=P_{v} \quad K_{5}=y P_{v}+2(1-\mu) u P_{y}-2 u^{1-\mu} P_{z}
\end{array}\right.
$$

whereas the 6 th one has a very special form [3] for $\mu=1 / 2$. One has

$$
\begin{cases}\mu \neq 1 / 2 & K_{6}=z P_{v}-2 u^{1-\mu} P_{y}-\frac{(2-\mu)}{(2 \mu-1)} u^{1-2 \mu} P_{z} \\ \mu=1 / 2 & K_{6}=z P_{v}-2 \sqrt{u} P_{y}+\frac{3}{2} \ln (u) P_{z} .\end{cases}
$$

Keane and Tupper have shown that the tensor

$$
S_{\mu \nu}=k_{(\mu} Y_{\nu)}^{H}+\mu u g_{\mu \nu}
$$

is indeed K-S. However one can check that for $\mu \neq 1 / 2$ the corresponding conserved quadratic quantity is fully reducible

$$
S^{\mu \nu} P_{\mu} P_{\nu}=-K_{1} K_{4}+K_{2} K_{5}+(1-2 \mu) K_{3} K_{6}
$$

as well as for $\mu=1 / 2$

$$
S^{\mu \nu} P_{\mu} P_{\nu}=-K_{1} K_{4}+K_{2} K_{5}+\frac{3}{2} K_{3}^{2}
$$

We conclude to the full reducibility of this K-S tensor in all cases, in agreement with the Levi-Civita conditions. 


\section{No hyperkähler metrics except for type III}

We have seen that for Bianchi type III non-diagonal hyperkähler metrics do exist. This is very special to type III since one has the following:

Proposition 4 Among Bianchi B empty space metrics (excluding type III) there are no hyperkähler metrics.

Proof: For the ease of the reader we will split up the proof into three steps.

Step 1: the self-dual spin connection

Let us write the metric as

$$
g=d t^{2}+\gamma_{i j}(t) \sigma_{i} \sigma_{j} \quad i, j=1,2,3
$$

where the matrix $\gamma$ can be taken symmetric and must be positive definite and invertible. The Maurer-Cartan invariant 1-forms have for differentials

$$
d \sigma_{i}=\frac{1}{2} C_{i ; s t} \sigma_{s} \wedge \sigma_{t}
$$

or explicitly for the type B Lie algebras

$$
d \sigma_{1}=0 \quad d \sigma_{2}=n_{2} \sigma_{3} \wedge \sigma_{1}+a \sigma_{1} \wedge \sigma_{2} \quad d \sigma_{3}=-a \sigma_{3} \wedge \sigma_{1}+n_{3} \sigma_{1} \wedge \sigma_{2}
$$

with the table

$\begin{array}{cccrl} & a & n_{2} & n_{3} & \\ \text { type } I I I & 1 & 1 & -1 & \\ \text { type } I V & 1 & 0 & 1 & \\ \text { type } V & 1 & 0 & 0 & \\ \text { type } V I_{h} & \sqrt{-h} & 1 & -1 & (h<0, h \neq-1) \\ \text { type } V I I_{h} & \sqrt{h} & 1 & -1 & (h>0)\end{array}$

It is well known that one can find some invertible symmetric matrix $\rho$ such that $\gamma=\rho^{t} \rho$. So it is convenient to define the tetrad

$$
e_{0}=d t \quad e_{i}=\rho_{i j}(t) \sigma_{j} \quad i, j=1,2,3,
$$

and some algebra gives for the self-dual components of the spin connection

$$
\omega_{i}^{+} \equiv \omega_{0 i}+\frac{1}{2} \epsilon_{i j k} \omega_{j k}=g_{i}(t) d t+m_{i j}(t) e_{j}
$$

where

$$
m_{i j}=L_{i j}-(\dot{\rho} r)_{(i j)} \quad g_{i}=-\epsilon_{i j k}(\dot{\rho} r)_{[j k]} \quad r=\rho^{-1}
$$

and

$$
L_{i j}=\frac{1}{2 \operatorname{det} \rho}\left(\operatorname{Tr}(\rho \mu \rho) \delta_{i j}-2(\rho \mu \rho)_{i j}\right), \quad \mu_{i j}=\frac{1}{2} \epsilon_{i s t} C_{j ; s t} \rightarrow\left(\begin{array}{rrr}
0 & 0 & 0 \\
0 & n_{2} & -a \\
0 & a & n_{3}
\end{array}\right)
$$


Let us notice that the matrix $L$ is never diagonal. Its skew-symmetric part is

$$
L_{[i j]}=a \epsilon_{i j k} r_{1 k} \quad r=\rho^{-1} .
$$

So if we impose $\omega_{i}^{+}=0$ we must have $L_{[i j]}=0$ and since for all Bianchi type B Lie algebras $a \neq 0$, this implies that $r_{1 k}=0$ for $k=1,2,3$ contradicting the hypothesis that $\rho$ is invertible. So there are no hyperkähler Bianchi B metrics with vanishing self-dual connection.

Step 2: the self-dual curvature

Recalling the triplet of 2-forms with self-dual curvature

$$
R_{i}^{+}=d \omega_{i}^{+}-\frac{1}{2} \epsilon_{i j k} \omega_{j}^{+} \wedge \omega_{k}^{+}
$$

an easy computation shows that

$$
R_{i}^{+}=0 \quad\left\{\begin{array}{ll}
\dot{M}_{i j}=\epsilon_{i s t} g_{s} M_{t j} & (a) \\
M_{i j} C_{j ; s t}=\epsilon_{i j k} M_{j s} M_{k t} & (b)
\end{array} \quad M=m \rho .\right.
$$

Differentiating (104)(b) and using (104)(a) gives nothing but an identity.

Step 3: structure of the matrix $M$ and conclusion

Writing the matrix $M$ in terms of its column vectors

$$
M=\left(M_{1} M_{2} M_{3}\right)
$$

the relations (104)(b) split up into the system

$$
\left\{\begin{array}{l}
A M_{2}=a M_{2}+n_{3} M_{3} \\
A M_{3}=-n_{2} M_{2}+a M_{3} \quad A_{i j}=\epsilon_{i s j} M_{s 1} . \\
0=\epsilon_{i j k} M_{j 2} M_{k 3}
\end{array}\right.
$$

Using the table (98) and observing that the skew-symmetric matrix $A$ may have only 0 as a real eigenvalue, it is easy to prove that we must have $M_{2}=M_{3}=0$. This fails to be true for the type III.

Denoting by $b_{1}, b_{2}, b_{3}$ the components of the column vector $M_{1}$, we can compute the skew-symmetric part of the matrix $m=M r$ and by the first relation in (101) we must have $m_{[i j]}=L_{[i j]}$. This gives a linear system

$$
\begin{array}{r}
b_{2} r_{11}-b_{1} r_{12}+2 a r_{13}=0 \\
2 a r_{11}+b_{3} r_{12}-b_{2} r_{13}=0 \\
-b_{3} r_{11}+2 a r_{12}+b_{1} r_{13}=0
\end{array}
$$

the determinant of which is $2 a\left(b_{1}^{2}+b_{2}^{2}+b_{3}^{2}+4 a^{2}\right) \neq 0$. This implies that $r_{11}, r_{12}$ and $r_{13}$ do vanish, contradicting the hypothesis that $\rho$ must be invertible.

This ends up the proof.

This Proposition implies that there are not even flat euclidean metrics, since these would be hyperkähler. 


\section{Conclusion}

We hope to have clarified and simplified some aspects of the analysis developed in [3] and [4. In our opinion the most striking discovery of Christodoulakis and Terzis is that, for most of the Bianchi B metrics, the (empty space) Einstein equations do exhibit the Painlevé property no matter what the signature be. Such a phenomenon was observed earlier for the diagonal metrics of types $\mathrm{VI}_{0}$ and $\mathrm{VII}_{0}$ : Lorenz-Petzold [13] reduced them to Painlevé III. This shows that the theoretical prejudice according to which this should happen rather for euclidean metrics with self-dual Weyl tensor is far from being the whole story.

Certainly nobody would bet that the empty space Einstein equations do enjoy in general the Painlevé property, however this may happen for particular classes of metrics! So it would be of great interest to have an answer to the question: which classes of metrics do exhibit the Painlevé property? In view of the formidable difficulty of this question its answer is probably "the stuff the dreams are made of".

\section{References}

[1] J. E. Åman, "Classical general relativity" (Proceedings of the 1983 London conference), 1-4, ed. W.B. Bonnor, J.N. Islam and M.A.H. MacCallum, Cambridge University Press (1983).

[2] M. Cahen and L. Defrise, Commun. Math. Phys.,11 (1968) 56.

[3] T. Christodoulakis and P. A. Terzis, J. Math. Phys., 47 (2006) 102502.

[4] T. Christodoulakis and P. A. Terzis, Class. Quant. Grav., 24 (2007) 875.

[5] C. D. Collinson, Int. J. Theor. Phys., 15 (1976) 311.

[6] C. M. Cosgrove and G. Scoufis, Stud. Appl. Math., 88 (1993) 25.

[7] C. Duval and G. Valent, J. Math. Phys., 46, 053516 (2005).

[8] R. Geroch, J. Math. Phys., 12 (1971) 1126.

[9] G. W. Gibbons and P. J. Ruback, Commun. Math. Phys., 115 (1988) 267.

[10] V. I. Gromak, I. Laine and S. Shimomura, Painlevé Differential Equations in the Complex Plane, Walter de Gruyter, Berlin New-York (2002).

[11] A. J. Keane and B. O. Tupper, Class. Quant. Grav., 27 (2010) 245011.

[12] W. Kinnersley, J. Math. Phys., 10 (1969) 1195.

[13] D. Lorenz-Petzold, Acta Phys. Polon., B 15 (1984) 117.

[14] M. A. H. MacCallum, A. Moussiaux, P. Tombal and J. Demaret, J. Phys A: Math. Gen., 15 (1982) 1757.

[15] A. Moussiaux, P. Tombal and J. Demaret, J. Phys A: Math. Gen., 14 (1981) L277. 
[16] S. T. C. Siklos, J. Phys. A: Math. Gen., 14 (1981) 395.

[17] K. Okamoto, Ann. Mat. Pura Appl. 146 (1987) 337.

[18] A. M. Perelomov, Integrable systems of Classical Mechanics and Lie Algebras, Birkhauser Verlag, Basel-Boston-Berlin (1990).

[19] J. M. Stewart and G. F. R. Ellis, J. Math. Phys., 9 (1968) 1072.

[20] H. Stephani, D. Kramer, M MacCallum, C. Hoenselaers and E. Herlt, Exact solutions of Einstein's field equations, 2nd edition, Cambridge University Press (2003).

[21] P. A. Terzis and T. Christodoulakis, Gen. Relativ. Gravit., 41 (2009) 469.

[22] P. A. Terzis and T. Christodoulakis, arXiv:gr-qc/1007.1561.

[23] G. Valent, Commun. Math. Phys., 244 (2004) 571.

[24] G. Valent and H. Ben Yahia, Class. Quantum Grav., 24 (2007) 255.

[25] M. Walker and R. Penrose, Commun. Math. Phys., 18 (1970) 265. 